\title{
Efficacy and safety of endoscopic ultrasound-guided radiofrequency ablation for management of pancreatic lesions: a systematic review and meta-analysis
}

\author{
Yazan Fahmawi ${ }^{1}$, Ansh Mehta ${ }^{1}$, Haneen Abdalhadi ${ }^{1}$, Lindsey Merritt ${ }^{2}$, Meir Mizrahi ${ }^{2}$ \\ ${ }^{1}$ Department of Internal Medicine, University of South Alabama, Mobile, AL, USA; ${ }^{2}$ Department of Gastroenterology and Hepatology, Advanced \\ Endoscopy Unit, University of South Alabama, Mobile, AL, USA \\ Contributions: (I) Conception and design: Y Fahmawi, M Mizrahi; (II) Administrative support: None; (III) Provision of study materials or patients: Y \\ Fahmawi; (IV) Collection and assembly of data: Y Fahmawi, A Mehta; (V) Data analysis and interpretation: Y Fahmawi, A Mehta; (VI) Manuscript \\ writing: All authors; (VII) Final approval of manuscript: All authors. \\ Correspondence to: Meir Mizrahi, MD. Assistant Professor of Gastroenterology and Hepatology, Director of Advanced Endoscopy Center, Advanced \\ Endoscopy Fellowship Program Director, 75 S. University boulevard, UCOM 6000 Mobile, AL 36688, USA. \\ Email: mmizrahi@health.southalabama.edu.
}

Background: Radiofrequency ablation (RFA) has been used to treat various abdominal tumors including pancreatic tumors. Multiple approaches such as laparoscopic, open, and percutaneous have been used for pancreatic tissue ablation. More recently, endoscopic ultrasound (EUS)-guided RFA has emerged as a new technique for pancreatic tissue ablation. The role of EUS-RFA in management of pancreatic lesions is still not well-established. In this study, our aim is to assess efficacy and safety of EUS-RFA for management of pancreatic lesions.

Methods: MEDLINE, Scopus, and Cochrane Library databases were searched to identify studies reporting EUS-RFA of pancreatic lesions with outcomes of interest. Studies with $<5$ patients were excluded. Clinical success was defined as symptom resolution, decrease in tumor size, and/or evidence of necrosis on radiologic imaging. Efficacy was assessed by the pooled clinical response rate whereas safety was assessed by the pooled adverse events rate. Heterogeneity was assessed using $\mathrm{I}^{2}$. Pooled estimates and the $95 \% \mathrm{CI}$ were calculated using random-effect model.

Results: Ten studies ( 5 retrospective and 5 prospective) involving 115 patients with 125 pancreatic lesions were included. 152 EUS-RFA procedures were performed. The lesions comprised of $37.6 \%$ non-functional neuroendocrine tumors (NFNETs), 15.4\% were insulinomas, 26.5\% were pancreatic cystic neoplasms (PCNs), and $19.7 \%$ were pancreatic adenocarcinomas. The majority were present in the pancreatic head (40.2\%), $38.3 \%$ in the body, $11.2 \%$ in the tail, and $10.3 \%$ in the uncinate process. Pooled overall clinical response rate was $88.9 \%$ (95\% CI: 82.4-93.7, $\mathrm{I}^{2}=38.1 \%$ ). Pooled overall adverse events rate was $6.7 \%$ (95\% CI: $3.4-11.7, \mathrm{I}^{2}=34.0 \%$ ). The most common complication was acute pancreatitis (3.3\%) followed by pancreatic duct stenosis, peripancreatic fluid collection, and ascites $(2.8 \%)$ each. Only one case of perforation was reported with pooled rate of $(2.1 \%)$.

Discussion: This study demonstrates that EUS-RFA is an effective treatment modality for pancreatic lesions, especially functional neuroendocrine tumors such as insulinomas.

Keywords: Radiofrequency ablation (RFA); pancreatic tumors; pancreatic neuroendocrine tumors; insulinomas; non-resectable pancreatic tumors

Received: 04 February 2020; Accepted: 16 June 2020; Published: 25 July 2022.

doi: $10.21037 /$ tgh-20-84

View this article at: http://dx.doi.org/10.21037/tgh-20-84 


\section{Introduction}

As a result of advances in cross sectional imaging, incidental pancreatic lesions are being diagnosed with increasing frequency (1). These consist of solid and cystic pancreatic tumors, including pancreatic neuroendocrine tumors, pancreatic adenocarcinoma, insulinoma and various pancreatic cystic neoplasms. Surgery, radiation and chemoradiation are generally the mainstay management of these lesions. Percutaneous, intraoperative, and laparoscopic radiofrequency ablation (RFA) have been used for the management of various tumors including hepatocellular carcinoma, liver metastasis, primary lung tumors, and pancreatic tumors (2). RFA mainly works by thermal energy induced coagulative necrosis. Additionally, some studies have reported development of $\mathrm{T}$ cell immunity against tumor antigens subsequent to RFA ablation (3). Pancreas is known to be thermosensitive, surrounded with vascular and biliary structures, making it a vulnerable target. Therefore, intraoperative or laparoscopic RFA for pancreatic tumor management has been associated with high adverse events rate. Transabdominal US guided RFA is less invasive but limits visualization of pancreas situated retroperitoneally. Endoscopic ultrasound-guided RFA (EUS-RFA) has emerged as a promising approach as it is less invasive, provides real time visualization and precision for RFA. Supplemented with doppler imaging, this also enables visualization of vascular structures. Recently, many studies have been done using EUS-RFA for pancreatic tumors management. However, these studies have reported varying results in term of safety and efficacy of EUS-RFA for pancreatic tumors management. Therefore, in this pooledanalysis study, our aim was to assess safety and efficacy of EUS-RFA for pancreatic tumor management. We present the following article in accordance with the PRISMA reporting checklist (available at https://tgh.amegroups.com/ article/view/10.21037/tgh-20-84/rc).

\section{Methods}

\section{Study selection, data extraction, and quality assessment}

A comprehensive literature search from inception until July 2019 of MEDLINE, Scopus, and Cochrane Library was conducted using the following keywords in different combination: "Endoscopic ultrasound", "Radiofrequency ablation", and "Pancreas". To increase the yield of our search strategy, the references of the included studies as well as the last three supplemental issues of Gastrointestinal
Endoscopy fournal were reviewed. Eligibility criteria were pre-determined by two authors (Y.F and A.M). Only studies in English reporting the clinical success and the adverse events rates were included. Clinical success was defined as symptoms resolution, decrease in tumor size, and/or evidence of necrosis on radiologic imaging. Given the rarity of the solid pancreatic tumors and EUS-guided RFA being an emerging new modality for solid pancreatic tumor management, moderate to high quality abstracts were included in this study. Studies involving 5 patients or less, animal and experimental studies, reviews and commentaries, were excluded. Studies were excluded as well if their data were included in a larger study or in a more recent study. All results were downloaded into EndNote X9 (Thompson ISI ResearchSoft, Philadelphia, Pennsylvania, USA). Any duplication was identified and removed. Titles and abstracts of the initially extracted articles were screened by two reviewers (Y.F and A.M). Both reviewers reviewed the full text of the potentially eligible studies. Any disagreement was resolved by consensus or by consulting a third author (M.M).

The data of interest from the included studies were extracted independently by two authors (Y.F and A.M) using a standardized excel sheet. The following data were extracted from each included study: study authors, publication year, study design, study location, patients demographics, lesions size and location, lesions types, clinical and imaging outcomes, number of RFA sessions/ procedures per lesion, number of RFA application per session per lesion, adverse events, type of RFA probe, and follow-up period. The extracted data from both reviewers were compared and any disagreement was resolved by consensus or by consulting a third author (M.M).

Two reviewers (Y.F and A.M) assessed independently the quality of the included studies using the Newcastle-Ottawa scale (NOS). Any disagreement was resolved by consensus or by consulting a third author (M.M). A score $>7$ was considered as a high-quality study, 4-6 was considered as moderate-quality study, and $<4$ was considered as a lowquality study.

\section{Statistical analysis}

EUS-RFA Efficacy was assessed by the pooled clinical success rate whereas safety was assessed by the pooled adverse events rate. For this meta-analysis, mild adverse events as such events are subjective and not reported uniformly across studies, were not included. We only 


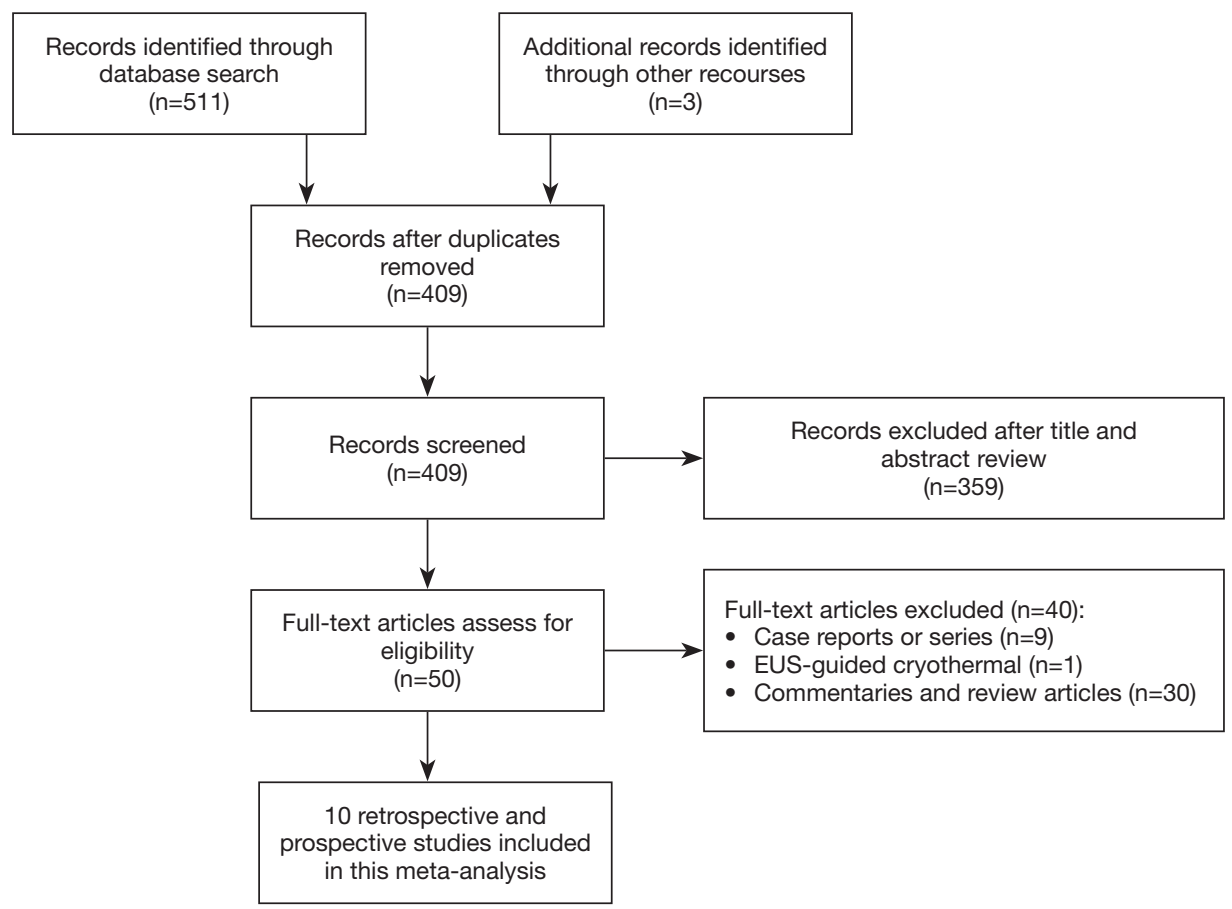

Figure 1 Study flow and selection. EUS, endoscopic ultrasound.

included serious adverse events that required subsequent intervention or resulted in prolonged hospitalization. Weighted pooled rate (WPR) along with $95 \%$ CI was calculated for the clinical success and adverse events rates. Heterogeneity was assessed by Cochrane Q test and $\mathrm{I}^{2}$ statistics. Presence of heterogeneity was considered if the Cochrane $\mathrm{Q}$ test was $\mathrm{P}<0.1$. Heterogeneity was considered substantial if $\mathrm{I}^{2}$ was $>50 \%$. Depending on the heterogeneity, random or fixed effects model was chosen. If the heterogeneity was substantial $(>50 \%)$, a random effect model was selected otherwise a fixed effects model was selected. WPR for adverse events were calculated out of total procedure number. Quantifying the publication bias if present was not done given the small number of the included studies. Publication bias was assessed by using the funnel plots for the clinical success and adverse events rates. Statistical analysis was performed using MedCalc software (Version 15.8).

\section{Results}

\section{Study characteristics and quality assessment}

Figure 1 depicts the selection process. 514 articles were identified from the search strategy and from manual search, of which 105 were duplicates. Of the remaining 409 articles, 359 were removed after screening the titles and the abstracts. After reviewing the remaining 50 articles, 10 articles met the inclusion criteria and were included in this study (1,4-12). The studies were conducted between 2015 and 2019 and 5 studies of them were prospective $(1,4,6,8,9)$ whereas the remaining 5 studies were retrospective (5,7,10-12). Of the 10 included studies, 3 of them were abstracts (10-12). EUS-RFA was planned to be performed on 115 patients with 125 pancreatic lesions. Table 1 shows baseline characteristics of the included studies. Table 2 shows indications as well as lesions locations in the included studies. Non-functional neuroendocrine pancreatic tumor (NFNET) was the most common lesion type (37.6\%), followed by pancreatic cystic neoplasms (26.5\%), unresectable pancreatic adenocarcinoma (19.7\%), and insulinomas (15.4\%). Most of the lesions were located in the head $(40.2 \%)$, followed by body (38.3\%), tail (11.2\%), and uncinate process $(10.3 \%)$. The quality of the included studies was assessed using the NOS scale. All the included studies were moderate in the methodological quality.

\section{Outcomes}

All of the included studies reported the outcomes of 
Table 1 Baseline characteristics of the included studies

\begin{tabular}{lcccccccc}
\hline Study & Country & Study design & $\begin{array}{c}\text { Patient } \\
\text { number }\end{array}$ & $\begin{array}{c}\text { Age, } \\
\text { years }\end{array}$ & Gender (M/F) & $\begin{array}{c}\text { Number of } \\
\text { lesions }\end{array}$ & $\begin{array}{c}\text { Lesion size } \\
\text { (mm) }\end{array}$ & $\begin{array}{c}\text { Study quality } \\
\text { using NOS }\end{array}$ \\
\hline Barthet 2019 & France & Prospective & 29 & 54.4 (mean) & $14 / 15$ & 31 & 21.3 (mean) & Moderate \\
Choi 2018 & South Korea & Prospective & 10 & 51.4 (mean) & $4 / 6$ & 10 & 17.5 (mean) & Moderate \\
Crino 2018 & Italy & Retrospective & 9 & 67 (mean) & $6 / 3$ & 8 & 36 (mean) & Moderate \\
Oleinikov 2019 & Israel & Retrospective & 18 & 60.4 (mean) & $10 / 8$ & 27 & 14.3 (mean) & Moderate \\
Pai 2015 & International & Prospective & 8 & 59.8 (mean) & $1 / 7$ & 8 & 34.25 (mean) & Moderate \\
Scopelliti 2018 & Italy & Prospective & 10 & 62 (median) & $7 / 3$ & 10 & Not reported & Moderate \\
Song 2016 & South Korea & Prospective & 6 & 60 (median) & $1 / 5$ & 6 & 4.9 (mean) & Moderate \\
Dancour 2019 & Israel & Retrospective & 8 & 58 (median) & $4 / 4$ & 8 & 16 (median) & Moderate \\
Yang 2019 & China & Retrospective & 8 & Not reported & Not reported & 8 & Not reported & Moderate \\
De la Serna 2018 & Spain & Retrospective & 9 & 69 (median) & 9 & 9 & 19.2 (mean) & Moderate \\
\hline
\end{tabular}

NOS, Newcastle-Ottawa scale.

Table 2 Indications and lesions sites of the included studies

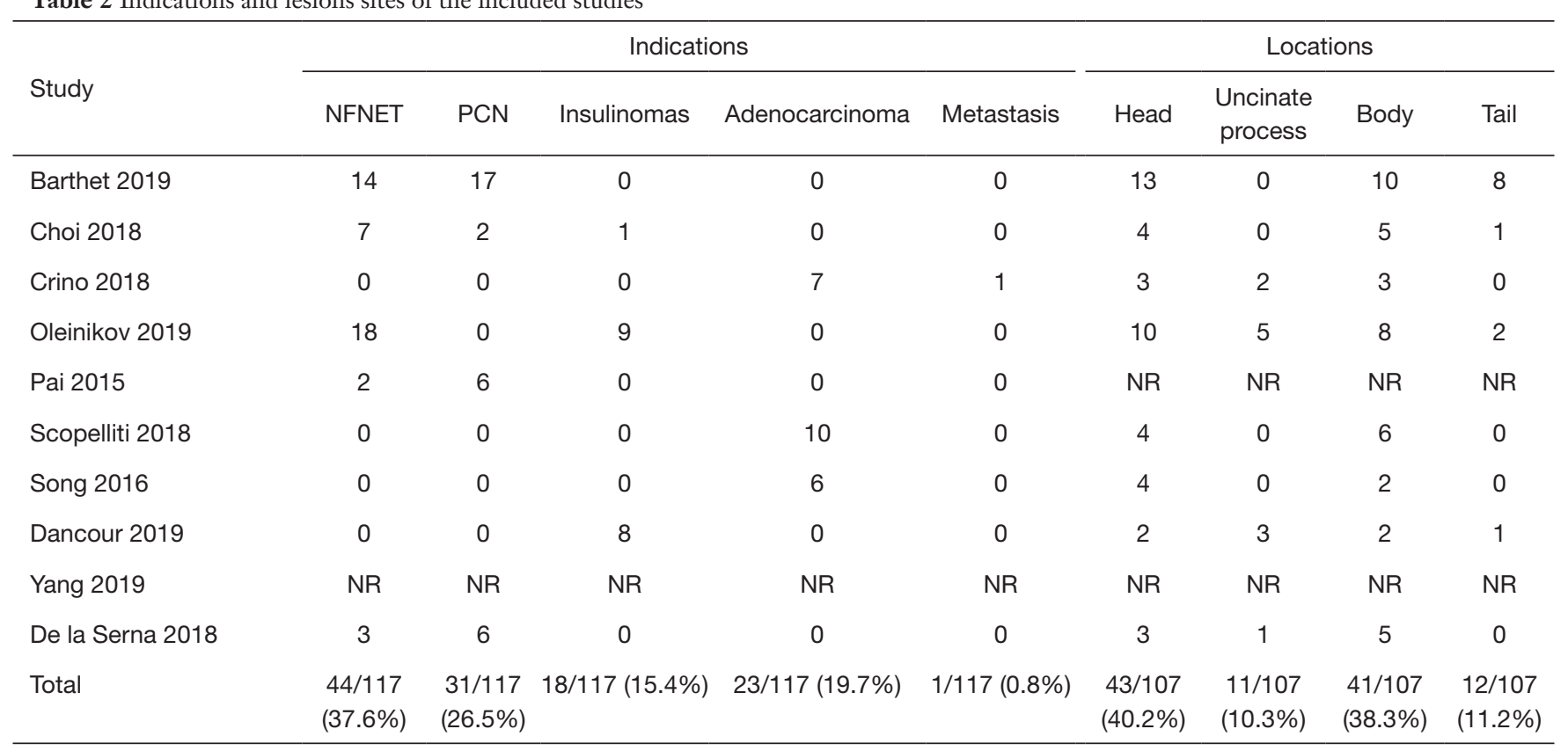

NFNET, non-functional neuroendocrine tumor; PCN, pancreatic cystic neoplasm; NR, not reported.

interest, clinical response, and adverse events (Tables 3,4). The WPR for clinical response was $88.9 \%$ (95\% CI: 82.4-93.7), with moderate heterogeneity, Cochran Q test $\mathrm{P}=0.10, \mathrm{I}^{2}=38.1 \%$ (Figure 2). Funnel plot for clinical response was symmetrical (Figure 3). Table 4 demonstrates the WPR for each pancreatic lesion type. The WPR for adverse events was $6.7 \%$ (95\% CI: 3.4-11.7), with moderate heterogeneity, Cochran $\mathrm{Q}$ test $\mathrm{P}=0.14, \mathrm{I}^{2}=34.0 \%$ (Figure 4). Funnel plot for adverse events was fairly symmetrical (Figure 5). The most common adverse event was acute pancreatitis with WPR of $(3.3 \%)$ whereas the WPR for pancreatic duct stenosis, peripancreatic fluid collection, and ascites, were (2.8\%) each. Perforation had WPR of (2.1\%). 


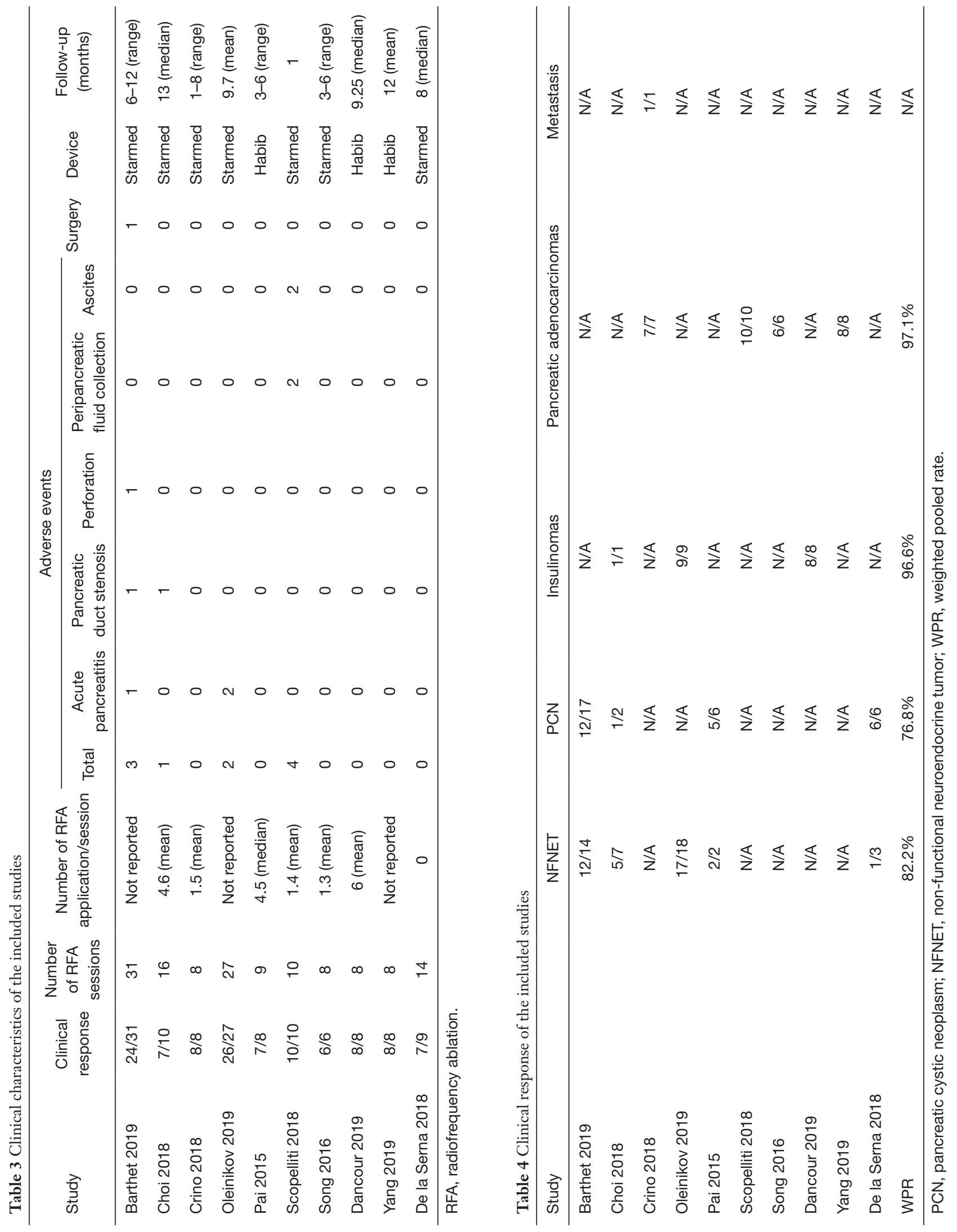




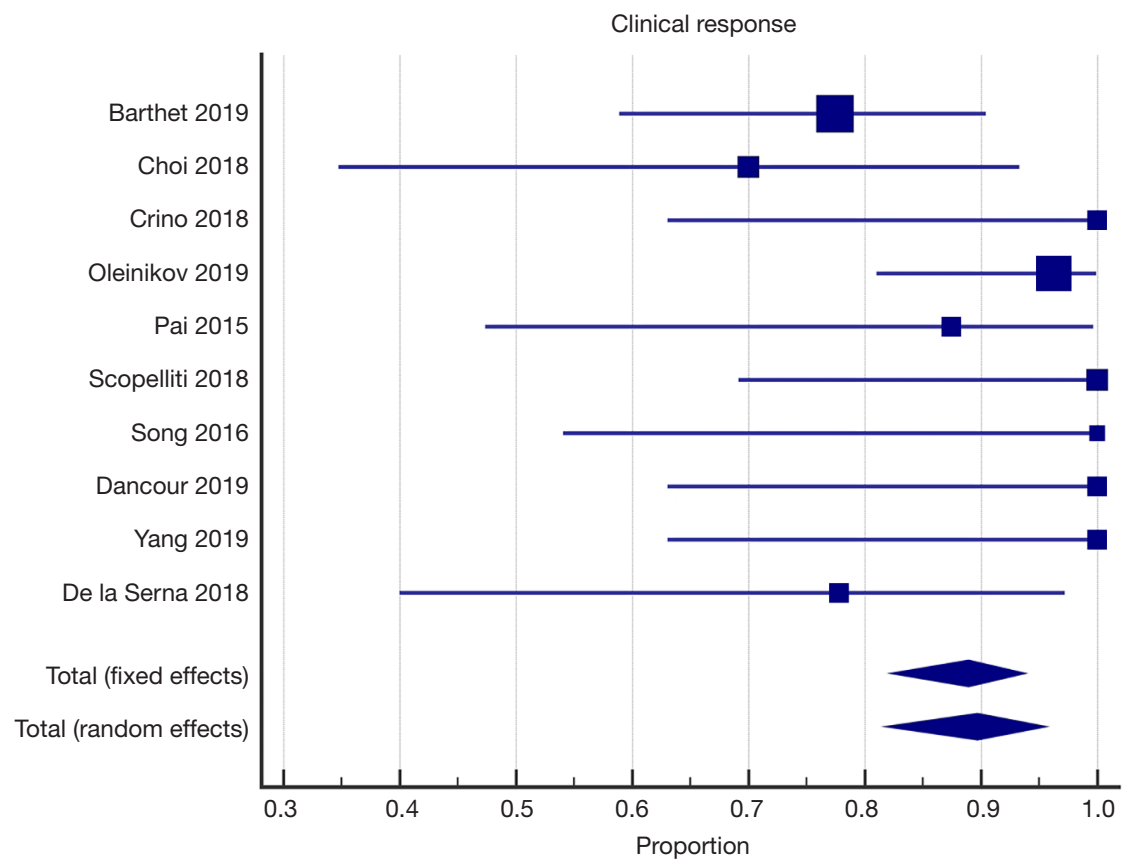

Figure 2 Forest plot for overall clinical response. Size of the square is proportional to the precision of the study-specific effect estimates, and the bars indicate the corresponding $95 \%$ CIs. The diamond is placed on the summary correlation coefficient of the observational studies, and the width indicates the corresponding $95 \%$ CI.

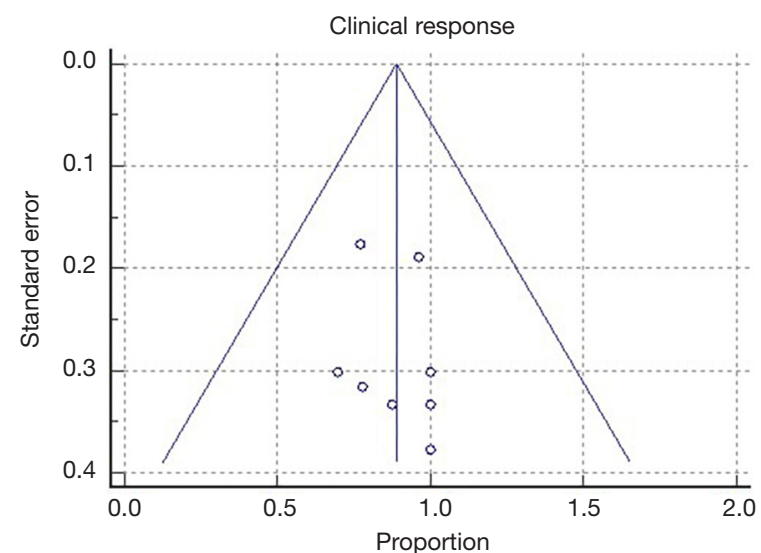

Figure 3 Funnel plot for overall clinical response.

\section{Discussion}

RFA has been used to treat various abdominal tumors including pancreatic tumors. Multiple approaches such as laparoscopic, open, and percutaneous have been used for pancreatic tissue ablation. More recently, EUS-RFA has emerged as a new technique for pancreatic tissue ablation, however the role of EUS-RFA in management of pancreatic lesions is still not well-established. Thereby, the aim of this study to evaluate the cumulative efficacy and safety of this new technique.

Given the thermosensitivity of the normal pancreatic tissue as well as its proximity to vital structures such as main pancreatic duct, common bile duct, and major blood vessels, both percutaneous and intra-operative RFA have been associated with technical difficulties and high adverse event rates. In addition to the former factors, the optimal thermal kinetic characteristics for the pancreas have not been determined, so there is still no standardized protocol for pancreatic RFA (13-15). In a study involving 50 patients with unresectable pancreatic cancer that underwent ultrasound guided RFA during laparotomy, the adverse events occurred in $24 \%$ of patients. At least half of these adverse events were attributed to RFA (16). In another study involving 10 patients who underwent RFA (7 percutaneous, 2 intraoperative, and 1 EUS-guided) for pancreatic neuroendocrine tumor, 3 patients developed acute pancreatitis with 2 of them complicated with peripancreatic fluid collection (17). In our study, the overall pooled adverse event rate was $6.7 \%$. The most common adverse event was acute pancreatitis with pooled rate of $3.3 \%$. Oleinikov et al. reported two cases of acute pancreatitis in his study while Barthet et al. reported one case of acute pancreatitis with 


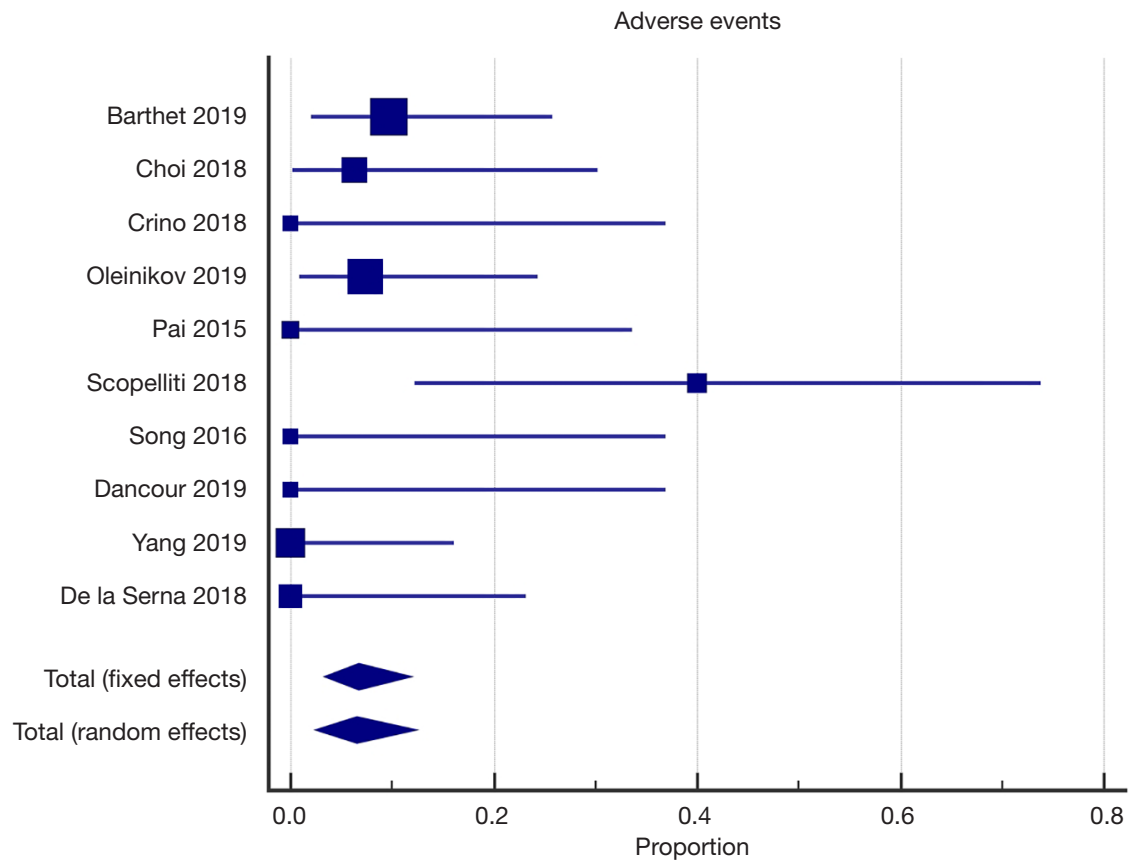

Figure 4 Forest plot of overall adverse events rate. Size of the square is proportional to the precision of the study-specific effect estimates, and the bars indicate the corresponding $95 \%$ CIs. The diamond is placed on the summary correlation coefficient of the observational studies, and the width indicates the corresponding 95\% CI.

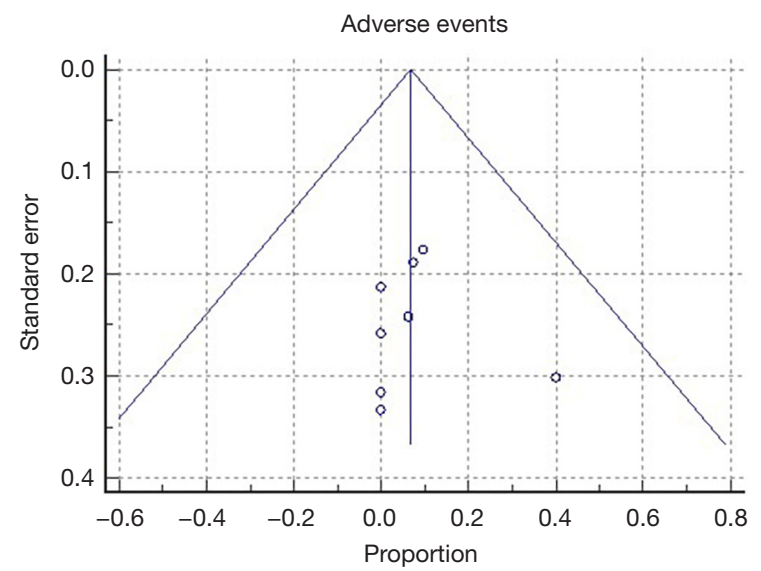

Figure 5 Funnel plot for overall adverse events rate.

early signs of infection, occurring early on in this study $(4,7)$. However, after initiating a prophylaxis protocol by administering rectal indomethacin and antibiotics, Barthet et al. reported no further cases of acute pancreatitis. Similarly, prophylactic antibiotics were given before the procedures in all of the included studies (5,7-9) except in two studies $(1,5)$. Interestingly, no adverse events were reported in those two studies. In addition to acute pancreatitis, pancreatic duct stenosis, peripancreatic fluid collection, and ascites complicated two procedures each with a pooled rate of $(2.8 \%)$ each. Only one procedure was complicated by a jejunal perforation that required surgical intervention. The procedure was performed for a cystic neoplasm without suctioning the cystic fluid. It is hypothesized that fluid inside the cyst might increase the impedance leading to requirement of higher thermal energy which may increase the risk of adverse events. Therefore, Barthet et al. suggest cystic drainage before applying RFA (4). Ultimately, EUS-RFA has a good safety profile. Applying prophylactic measures such as antibiotics, rectal indomethacin, and/or draining cystic lesions before EUS-RFA might help in decrease the risk of adverse events. Despite these promising results, large controlled prospective trials are warranted.

Regarding EUS-RFA efficacy for management of pancreatic tumors, this study demonstrates that EUSRFA has an excellent efficacy for solid pancreatic tumors management with pooled clinical response rate of $89.8 \%$. Importantly, although the heterogeneity was not substantial (39\%), there were differences in the indications as well as definitions of clinical response among the included studies.

Surgical resection is the treatment of choice for 
localized insulinomas. Other less invasive modalities for management of insulinomas such as EUS-guided alcohol ablation or embolization, have been described $(18,19)$. All of the insulinomas that were included in this metaanalysis responded well to EUS-RFA with resolution of the symptoms. The effect of EUS-RFA for insulinomas may not be instantaneous and can be delayed. Spontaneous resolution of symptoms after months of treating insulinomas with EUSRFA has been reported $(4,10)$. This delayed response is most likely attributed to stimulation of the immune system resulting in local and systemic antitumor activity (20). Thus, offering a new promising treatment modality for insulinoma management. Similar to insulinomas, all pancreatic adenocarcinomas responded with either necrosis or decrease in size on imaging in the included studies. Surgical resection is the only curative option for pancreatic adenocarcinoma. However, only $20 \%$ of patients present with a resectable disease. Like local ablation using RFA during open or laparoscopic surgery have shown improvement in overall survival in patients with unresectable pancreatic adenocarcinomas (16,21). Unlike insulinomas and unresectable pancreatic adenocarcinomas, not all NFNET had responded to EUS-RFA. Large ( $>2 \mathrm{~cm})$, symptomatic, involvement of main pancreatic duct, presence of resectable regional nodal metastases, or presence of synchronous resectable distant metastases, are indications for surgical resection of NFNET. However, the data about incidentally diagnosed, small, asymptomatic NFNET is still controversial $(22,23)$. The European Neuroendocrine Tumor Society recommends a "wait and see" approach for these small asymptomatic NFNET (22). However, some studies showed that many patients decline active surveillance with imaging and prefer to proceed with surgery $(24,25)$. Therefore, EUSRFA offers a minimally invasive treatment modality for small and asymptomatic NFNET as well as for patients at high risk for surgical intervention.

Pancreatic cystic neoplasms have become the most frequent premalignant pancreatic tumor encountered $(26,27)$. EUS-guided ablation using ethanol injection has been used to treat pancreatic cystic neoplasms. In a metaanalysis study conducted by Kandula et al., complete cyst resolution rate using EUS-guided ethanol ablation was $56 \%$ while partial resolution rate was $23.7 \%$. In the same study, acute pancreatitis rate following EUS-guided ethanol ablation was $3.9 \%$ (28). Although the clinical response was higher in our study (76.8\%), the definition of clinical response is different between our study and Kandula et al. study as we considered all types of response (complete resolution and partial resolution) in our study. The rate of acute pancreatitis between our study and Kandula et al. study was comparable (3.3\% and 3.9\%, respectively) (28). However, our study included different types of pancreatic lesions while Kandula et al. included only pancreatic cystic neoplasms.

In conclusion, EUS-RFA offers a safe and effective treatment modality for pancreatic lesions especially functional neuroendocrine tumors. Despite these promising results, this study does have a few limitations, including a small number of included patients. Additionally, the studies included studies were non-controlled cohort studies, with significant variations in the indications, techniques, and the definitions of clinical response.

\section{Acknowledgments}

Funding: None.

\section{Footnote}

Reporting Checklist: The authors have completed the PRISMA reporting checklist. Available at https://tgh. amegroups.com/article/view/10.21037/tgh-20-84/rc

Conflicts of Interest: All authors have completed the ICMJE uniform disclosure form (available at https://tgh.amegroups. com/article/view/10.21037/tgh-20-84/coif). The authors have no conflicts of interest to declare.

Ethical Statement: The authors are accountable for all aspects of the work in ensuring that questions related to the accuracy or integrity of any part of the work are appropriately investigated and resolved.

Open Access Statement: This is an Open Access article distributed in accordance with the Creative Commons Attribution-NonCommercial-NoDerivs 4.0 International License (CC BY-NC-ND 4.0), which permits the noncommercial replication and distribution of the article with the strict proviso that no changes or edits are made and the original work is properly cited (including links to both the formal publication through the relevant DOI and the license). See: https://creativecommons.org/licenses/by-nc-nd/4.0/.

\section{References}

1. Pai M, Habib N, Senturk H, et al. Endoscopic ultrasound 
guided radiofrequency ablation, for pancreatic cystic neoplasms and neuroendocrine tumors. World J Gastrointest Surg 2015;7:52-9.

2. Shah DR, Green S, Elliot A, et al. Current oncologic applications of radiofrequency ablation therapies. World J Gastrointest Oncol 2013;5:71-80.

3. Rustagi T, Chhoda A. Endoscopic Radiofrequency Ablation of the Pancreas. Dig Dis Sci 2017;62:843-50.

4. Barthet M, Giovannini M, Lesavre N, et al. Endoscopic ultrasound-guided radiofrequency ablation for pancreatic neuroendocrine tumors and pancreatic cystic neoplasms: a prospective multicenter study. Endoscopy 2019;51:836-42.

5. Crinò SF, D'Onofrio M, Bernardoni L, et al. EUS-guided Radiofrequency Ablation (EUS-RFA) of Solid Pancreatic Neoplasm Using an 18-gauge Needle Electrode: Feasibility, Safety, and Technical Success. J Gastrointestin Liver Dis 2018;27:67-72.

6. Choi JH, Seo DW, Song TJ, et al. Endoscopic ultrasoundguided radiofrequency ablation for management of benign solid pancreatic tumors. Endoscopy 2018;50:1099-104.

7. Oleinikov K, Dancour A, Epshtein J, et al. Endoscopic Ultrasound-Guided Radiofrequency Ablation: A New Therapeutic Approach for Pancreatic Neuroendocrine Tumors. J Clin Endocrinol Metab 2019;104:2637-47.

8. Scopelliti F, Pea A, Conigliaro R, et al. Technique, safety, and feasibility of EUS-guided radiofrequency ablation in unresectable pancreatic cancer. Surg Endosc 2018;32:4022-8.

9. Song TJ, Seo DW, Lakhtakia S, et al. Initial experience of EUS-guided radiofrequency ablation of unresectable pancreatic cancer. Gastrointest Endosc 2016;83:440-3.

10. Dancour A, Benson A, Epshtein J, et al. Su1223 endoscopic ultrasound guided radiofrequency ablation of insulinomas is safe and effective. Gastrointest Endosc 2019;89:AB323.

11. Yang J, Zhang XJGE. Tu1357 feasibility and safety of EUS-guided radiofrequency ablation in unresectable pancreatic cancer. Gastrointest Endosc 2019;89:AB588-9.

12. De la Serna C, Cimavilla M, Madrigal B, et al. Su1384 EUS-guided radiofrequency ablation (eus-rfa) for pancreatic neuroendocrine tumors and cystic mucinous neoplasms: a pilot study of safety, feasibility and efficacy. Gastrointest Endosc 2018;87:AB332-3.

13. Changela K, Patil R, Duddempudi S, et al. Endoscopic Ultrasound-Guided Radiofrequency Ablation of the Pancreatic Tumors: A Promising Tool in Management of Pancreatic Tumors. Can J Gastroenterol Hepatol 2016;2016:4189358.
14. Mohan H, Nicholson P, Winter DC, et al. Radiofrequency ablation for neuroendocrine liver metastases: a systematic review. J Vasc Interv Radiol 2015;26:935-42.e1.

15. Elias D, Baton O, Sideris L, et al. Necrotizing pancreatitis after radiofrequency destruction of pancreatic tumours. Eur J Surg Oncol 2004;30:85-7.

16. Girelli R, Frigerio I, Salvia R, et al. Feasibility and safety of radiofrequency ablation for locally advanced pancreatic cancer. Br J Surg 2010;97:220-5.

17. Rossi S, Viera FT, Ghittoni G, et al. Radiofrequency ablation of pancreatic neuroendocrine tumors: a pilot study of feasibility, efficacy, and safety. Pancreas 2014;43:938-45.

18. Okabayashi T, Shima Y, Sumiyoshi T, et al. Diagnosis and management of insulinoma. World journal of gastroenterology 2013;19:829-37.

19. Schnack C, Hansen C, Beck-Nielsen H, et al. [Treatment of insulinomas with alcoholic ablation]. Ugeskr Laeger 2012;174:501-2.

20. Slovak R, Ludwig JM, Gettinger SN, et al. Immunothermal ablations - boosting the anticancer immune response. J Immunother Cancer 2017;5:78.

21. Girelli R, Frigerio I, Giardino A, et al. Results of 100 pancreatic radiofrequency ablations in the context of a multimodal strategy for stage III ductal adenocarcinoma. Langenbecks Arch Surg 2013;398:63-9.

22. Falconi M, Eriksson B, Kaltsas G, et al. ENETS Consensus Guidelines Update for the Management of Patients with Functional Pancreatic Neuroendocrine Tumors and Non-Functional Pancreatic Neuroendocrine Tumors. Neuroendocrinology 2016;103:153-71.

23. Partelli S, Bartsch DK, Capdevila J, et al. ENETS Consensus Guidelines for Standard of Care in Neuroendocrine Tumours: Surgery for Small Intestinal and Pancreatic Neuroendocrine Tumours. Neuroendocrinology 2017;105:255-65.

24. Gaujoux S, Partelli S, Maire F, et al. Observational study of natural history of small sporadic nonfunctioning pancreatic neuroendocrine tumors. J Clin Endocrinol Metab 2013;98:4784-9.

25. Jung JG, Lee KT, Woo YS, et al. Behavior of Small, Asymptomatic, Nonfunctioning Pancreatic Neuroendocrine Tumors (NF-PNETs). Medicine 2015;94:e983.

26. Farrell JJ. Prevalence, Diagnosis and Management of Pancreatic Cystic Neoplasms: Current Status and Future Directions. Gut Liver 2015;9:571-89.

27. Tanaka M, Fernández-Del Castillo C, Kamisawa T, et al. Revisions of international consensus Fukuoka 
Page 10 of 10

guidelines for the management of IPMN of the pancreas. Pancreatology 2017;17:738-53.

28. Kandula M, Moole H, Cashman M, et al. Success of

doi: $10.21037 / \operatorname{tgh}-20-84$

Cite this article as: Fahmawi Y, Mehta A, Abdalhadi H, Merritt L, Mizrahi M. Efficacy and safety of endoscopic ultrasound-guided radiofrequency ablation for management of pancreatic lesions: a systematic review and meta-analysis. Transl Gastroenterol Hepatol 2022;7:30. endoscopic ultrasound-guided ethanol ablation of pancreatic cysts: a meta-analysis and systematic review. Indian J Gastroenterol 2015;34:193-9. 